\title{
PHANTOM LIMB PAIN INDUCED BY SPINAL ANESTHESIA
}

André Prato Schmidt, Márcia Emy Takahashi, and Irimar de Paula Posso

Phantom pain, a neuropathic pain resulting from functional changes in peripheral and central pain pathways subsequent to amputation, is challenging to treat. ${ }^{1}$ It is a common sequela of amputation, occurring in up to $80 \%$ of patients who undergo the procedure. ${ }^{2}$ It must be differentiated from nonpainful phantom phenomena, residual-limb pain, and nonpainful residual-limb phenomena. Central changes seem to be a major determinant of phantom-limb pain, but peripheral and psychological factors may contribute to it. ${ }^{2}$

There have been reports that spinal anesthesia induces severe lightning pain in the lower limbs of patients with phantom limb pain, tabes dorsalis, or causalgia. ${ }^{3-6}$ The exact mechanism of this bizarre phenomenon is controversial. Some advocate that complete loss of sensory input after spinal anesthesia may decrease the level of inhibition and increase self-sustained neural activity. ${ }^{3}$

This report describes the onset of severe phantom pain following spinal anesthesia in a patient with an above-knee amputation of the left lower limb. We also discuss some potential mechanisms and management alternatives: A 72year-old man, $75 \mathrm{~kg}$, who suffered traumatic amputation of the left lower limb in December 1980, developing severe phantom pain after 3 months was described. Since 1998, he had had complete resolution of pain with carbamazepine $200 \mathrm{mg} \mathrm{q12}$ h. In March 2004, he was scheduled for surgical treatment of urinary incontinence. Relevant issues in his medical history included smoking (40 packs per year) and respiratory symptoms consistent with mild chronic obstrutive pulmonary disease. He was taking only oral carbamazepine, had no allergies, and no history of cardiovascular or gastrointestinal disease.

Anesthesiology Department, Hospital das Clínicas, Faculty of Medicine, University of São Paulo - São Paulo/SP, Brazil.

E-mail: aschmidt@ufrgs.br
He had undergone 2 surgical procedures previously: lower limb amputation in December 1980 and radical prostatectomy for prostate cancer in January 2003, both under general anesthesia, without complication.

His physical examination revealed: (respiratory system) increased anteroposterior diameter and occasional wheezing. Cardiovascular, abdominal, and neurologic examinations were unremarkable. A left supra-genicular amputation was noticed, presenting a well-healed scar, with no signs of infection or trigger zones.

In the operating room, routine monitoring was applied, and an 18-G catheter was inserted into left cephalic vein. After the infusion of $500 \mathrm{~mL}$ of lactated Ringer's solution, with the patient in the sitting position, a 27-G Whitacre spinal needle was introduced into the L3-4 interspace with no paresthesias. Hyperbaric bupivacaine (12.5 mg - 0.5\%) plus fentanil $(25 \mu \mathrm{g})$ were administered intrathecally. One minute after spinal anesthesia, the patient reported a severe burning pain sensation in his anatomically absent left lower limb, very similar to his previously phantom pain (visual analogue pain scale [VAS] of 10) accompanied by spontaneous contractions of his thigh. To treat this neuropathic pain, we performed an intravenous injection of fentanyl $100 \mu \mathrm{g}$ with good control of the pain sensation (VAS = 3). Moderate drowsiness was the only apparent complication. Analgesia to pin prick was attained to the T8 level, and the surgery proceeded without complication. After 2 hours, the patient was taken to the recovery room where regression of spinal blockade was accompained by complete resolution of pain (VAS $=0$ ). The patient was discharged 2 days later, and the follow-up revealed complete resolution of the phantom limb pain.

This patient presented a typical acute crisis of phantom pain related to a damaged nervous system as confirmed by clinical assessment. It was triggered by spinal anesthesia 6 years after the last crisis. 
The mechanism mediating phantom pain and its exacerbation by regional anesthesia is still unclear. ${ }^{7}$ The etiology of such a condition may be related to a peripheral or central origin, either spinal or cerebral. ${ }^{4}$ Such pain may be generated from active spinal cord cells that are released from inhibitory control through the loss of afferent impulses. ${ }^{8}$ After deafferentation, neuronal activity in the affected area of the spinal cord is characterized by spontaneous high-frequency burst activity. ${ }^{9}$ These neurons are usually inhibited by somatic sensory input and by projections from reticular formation. ${ }^{10}$ Loss of segmental afferent input due to regional anesthesia may decrease the central inhibitory effects on sensory transmission. The release from descending inhibition allows free transmission of abnormal bursting activity, evidenced by exacerbation of phantom limb pain. ${ }^{7}$ Counter irritation, either mechanical by percussion or electrical, may produce partial or total relief by increasing the level of inhibitory input. ${ }^{4}$ Risk factors for exacerbation of phantom pain after regional anesthesia are poorly understood, since there are few studies addressing this issue..$^{4,711,12}$ In patients with diabetes and leprosy, spinal instead of epidural anesthesia may constitute risk factors. ${ }^{4,7,11}$ In 17 patients with previous lower limb amputations who underwent spinal anesthesia who were studied prospectively, no risk factors were found. ${ }^{12}$ Since risk factors are not well studied, no preventive steps are available.

Phantom limb pain has been found to be extremely difficult to treat, and pain exacerbated by regional anesthesia may also be refractory to treatment with conventional drugs..$^{13}$ In previous reports, benzodiazepines, opioids, bar- biturates, calcitonin, neuroleptics, sodium channel blockers, volatile anesthetics, and N-methyl-D-aspartate (NMDA)receptor antagonists have been tried with variable results. ${ }^{4,7,14,15}$ Generally, drugs that suppress the central nervous system are thought to provide pain relief against newly developed pain after regional anesthesia. Intravenous thiopental $(1 \mathrm{mg} / \mathrm{kg})$ was thought to provide the best pain relief since it stopped the pain quickly, the dose was subanesthetic, and there was no adverse effect. ${ }^{7,10}$ Recently, opioids have been gaining acceptability for the management of neuropathic pain syndromes, mainly for those that are refractory to conventional treatment. ${ }^{15}$ Opioids are not descibed as the main-stay treatment for phantom limb pain after spinal anesthesia, but represent a well accepted alternative to thiopental, since they are safe and effective. ${ }^{10}$ In this case, we administered intravenous fentanyl, which was quite effective in this setting, as it has been in previous reports. ${ }^{10,15}$

In conclusion, spinal anaesthesia in amputees may cause reappearance of phantom pain in previously pain-free patients despite complete sensory anesthesia. However, few studies addressing risk factors or treatment are available. We suggest that additional well controlled studies addressing these issues are still needed. These studies should include larger sample sizes, homogenous methods for pain assessment, and long-term follow-up. To date, given the low incidence of phantom pain related to spinal anesthesia, the efficacy of treatment alternatives, and its transient nature, we conclude that spinal anaesthesia is not contraindicated in patients with previous lower limb amputation.

\section{REFERENCES}

1. Millan MJ. The induction of pain: an integrative review. Progress in Neurobiology. 1999;57:1-164.

2. Flor H. Phantom-limb pain: characteristics, causes, and treatment. Lancet Neurol. 2002;1(3):182-9.

3. Mackenzie N. Phantom limb pain during spinal anaesthesia. Recurrence in amputees. Anaesthesia. 1983;38(9):886-7.

4. Sellick BC. Phantom limb pain and spinal anesthesia. Anesthesiology. 1985;62(6):801-2.

5. Rowbottom SJ. Phantom pain and regional anaesthesia. Anaesth Intensive Care. 2000;28(3):337.

6. Sugiyama D, Nobuhara K, Maekawa N, Obara H. Severe lightning pain after spinal anesthesia in a patient with tabes dorsalis. Anesth Analg. 1989;69(2):268-9.

7. Fiddler DS, Hindman BJ. Intravenous calcitonin alleviates spinal anesthesia-induced phantom limb pain. Anesthesiology. 1991;74(1):187-9.

8. Sklar GS, Patel PR, Patel HJ, Kaplan LI. Phantom phenomena and anesthetically induced proprioceptive block. Reg Anesth. 1980;5:14-5.
9. Loeser JD, Ward AA, White LE. Chronic deafferentation of human spinal cord neurons. J Neurosurg. 1968;29:48-50.

10. Melzack R, Loeser JD. Phantom body pain in paraplegics: evidence for a central origin "pattern gererating mechanism" for pain. Pain. 1978;4:195-210.

11. Kato M, Tada F, Abe T, Okada T, Mori K, Go R, Kohyama A. Severe lightning pain during spinal anesthesia in a patient with diabetic neuropathy. Masui. 2000;49(9):1021-3.

12. Tessler MJ, Kleiman SJ. Spinal anaesthesia for patients with previous lower limb amputations. Anaesthesia. 1994;49(5):439-41.

13. Schmidt AP, Schmidt SRG. How effective are opioids in relieving neuropathic pain? The Pain Clinic. 2002;14(3):183-93.

14. Koyama K, Watanabe S, Tsuneto S, Takahashi H, Naito H. Thiopental for phantom limb pain during spinal anesthesia. Anesthesiology. 1988;69(4):598-600.

15. Wajima Z, Shitara T, Inoue T, Ogawa R. Severe lightning pain after subarachnoid block in a patient with neuropathic pain of central origin: Which drug is best to treat the pain? Clin J Pain. 2000;16(3):265-9. 